\title{
Teaching Nano-Thermodynamics: Gibbs Energy of Single-Component Nanoparticles
}

\author{
Jindřich Leitner ${ }^{1, *}$, David Sedmidubský ${ }^{2}$ \\ ${ }^{1}$ Department of Solid State Engineering, University of Chemistry and Technology, Prague, Czech Republic \\ ${ }^{2}$ Department of Inorganic Chemistry, University of Chemistry and Technology, Prague, Czech Republic \\ *Corresponding author: leitnerj@vscht.cz
}

\begin{abstract}
Much attention has been paid to thermodynamic modeling of nanosystems. A common approach consists in addition of a surface/interface term to the Gibbs energy of bulk materials and application of general conditions of equilibrium. Some discrepancy still remains dealing with the expression for surface contribution to molar Gibbs energy and chemical potential of components. It is shown, that due to the nonextensive nature of the surface area, these contributions are different for molar and partial molar quantities. The consistent expressions for the molar Gibbs energy and chemical potential of a single-component spherical nanoparticle are put forward along with the simple derivation of the Kelvin and Gibbs-Thomson equations.
\end{abstract}

Keywords: nanoparticle, Gibbs energy, chemical potential, Kelvin equation, Gibbs-Thomson equation

Cite This Article: Jindřich Leitner, and David Sedmidubský, "Teaching Nano-Thermodynamics: Gibbs Energy of Single-Component Nanoparticles.” World Journal of Chemical Education, vol. 5, no. 6 (2017): 206-209. doi: 10.12691/wjce-5-6-4.

\section{Introduction}

Recently, much attention has been paid to calculation of phase diagrams of nanoalloys $[1,2,3,4,5]$ as well as calculation of equilibrium constants of chemical reactions in nanosystems $[6,7,8,9]$. A common approach consists in addition of a surface/interface term to the Gibbs energy of bulk materials and application of general conditions of equilibrium in a closed system at constant temperature and pressure, namely equality of chemical potentials of individual components in coexisting phases or zero Gibbs energy of reaction. Surprisingly, some discrepancy still remains dealing with the expression of chemical potential of the involved components. Two quite similar but diverse terms are used for the surface contribution to Gibbs energy of spherical nanoparticles of radius $r$, namely

$$
\frac{2 \gamma V_{\mathrm{m}}}{r} \text { or } \frac{3 \gamma V_{\mathrm{m}}}{r} .
$$

This apparent controversy has been a subject of discussion and has been referred in a number of recently published papers [10-15]. The purpose of our contribution is to correctly derive relations for the molar Gibbs energy and chemical potential of single-component spherical nanoparticle and explain this apparent discrepancy. Moreover, the relation for chemical potential of nanoparticle is used for simple elucidation of the Kelvin and Gibbs-Thomson equations.

\section{Homogeneous Functions}

First, we recall the fundamental theorems about homogeneous functions for a better understanding of further explanation. A function $F(x)$ of variable $x$ is called the homogeneous function of the $k$-degree if it holds

$$
F(a x)=a^{k} F(x) .
$$

The Euler theorem gives for any homogeneous function of the $k$-degree the relation [16]

$$
F(x)=\frac{1}{k} x \frac{\mathrm{d} F(x)}{\mathrm{d} x} .
$$

For the homogeneous functions of the first order, and only for them, the relationship (2) results in

$$
\frac{F(x)}{x}=\frac{\mathrm{d} F(x)}{\mathrm{d} x} .
$$

\section{Gibbs Energy of Single-component Nanoparticles}

\subsection{Total Gibbs Energy of Nanoparticle}

Suppose a spherical nanoparticle of radius $r$ consisting of $n$ moles of the given substance. The total Gibbs energy of this particle $G^{\text {np }}$ can be express as

$$
G^{\mathrm{np}}=G^{\mathrm{b}}+G^{\mathrm{s}}=n G_{\mathrm{m}}^{\mathrm{b}}+\gamma A
$$

The superscripts b and s stand for the bulk and surface terms, respectively, $G_{\mathrm{m}}^{\mathrm{b}}$ is the molar Gibbs energy of the bulk, $\gamma$ denotes the surface energy (a reversible work that must be exerted to create a new surface/interface of unit area) and $A$ is the total surface/interface area. The bulk contribution $G^{\mathrm{b}}$ is a homogeneous function of the first 
order of the amount $n$ (extensive properties) [16] and it holds

$$
\frac{\mathrm{d} G^{\mathrm{b}}}{\mathrm{d} n}=\frac{G^{\mathrm{b}}}{n} \text {. }
$$

The surface contribution to the Gibbs energy of a spherical nanoparticle of radius $r$ can be expressed as

$$
\begin{aligned}
G^{\mathrm{S}} & =\gamma A=\gamma\left(4 \pi r^{2}\right)=\gamma 4 \pi\left(\frac{3 V}{4 \pi}\right)^{2 / 3} \\
& =\gamma 4 \pi\left(\frac{3 V_{\mathrm{m}}}{4 \pi}\right)^{2 / 3} n^{2 / 3}=C n^{2 / 3}
\end{aligned}
$$

where $C=\gamma(4 \pi)^{1 / 3}\left(3 V_{\mathrm{m}}\right)^{2 / 3}$ and $V_{\mathrm{m}}$ stands for molar volume. It follows from eq. (6) that the surface contribution to Gibbs energy is not an extensive property $[17,18,19]$ but a homogeneous function of the order $2 / 3$ (degree of homogeneity $k=2 / 3$ ) of the amount $n$. Hence it yields, according to the Euler theorem (2), the relation

$$
\frac{\mathrm{d} G^{\mathrm{S}}}{\mathrm{d} n}=\frac{2}{3} \frac{G^{\mathrm{S}}}{n} .
$$

The total Gibbs energy of the nanoparticle is not a homogeneous function of the amount $n$ as the condition (1) is not met.

\subsection{Molar Gibbs Energy of Nanoparticle}

The molar Gibbs energy of a spherical single-component nanoparticle of radius $r$ is given as

$$
\begin{aligned}
& G_{\mathrm{m}}^{\mathrm{np}}=\frac{G^{\mathrm{np}}}{n}=\frac{G^{\mathrm{b}}}{n}+\frac{G^{\mathrm{s}}}{n} \\
& =G_{\mathrm{m}}^{\mathrm{b}}+\gamma \frac{A}{n}=G_{\mathrm{m}}^{\mathrm{b}}+\gamma V_{\mathrm{m}} \frac{A}{V} .
\end{aligned}
$$

Based on the simple geometrical relations valid for a spherical nanoparticle of radius $r$

$$
A=4 \pi r^{2}, \quad V=\frac{4}{3} \pi r^{3}, \quad \frac{A}{V}=\frac{3}{r} .
$$

the expression for the molar Gibbs energy is given as

$$
G_{\mathrm{m}}^{\mathrm{np}}=G_{\mathrm{m}}^{\mathrm{b}}+\frac{3 \gamma V_{\mathrm{m}}}{r} .
$$

\subsection{Partial Molar Gibbs Energy (Chemical Potential) of Nanoparticle}

The chemical potential (partial molar Gibbs energy) of a spherical single-component nanoparticle of radius $r$ is given as a partial derivative of Gibbs energy $G$ with respect to substance amount $n$ at constant temperature and pressure. It holds for the bulk contribution ${ }^{1}$

\footnotetext{
${ }^{1}$ Constant pressure $p^{\text {in }}$ within a particle is considered here which is, due to the surface stress $f$, higher than the pressure $p$ in the surroundings. The condition of constant $p^{\text {in }}$ means such a variation of the surroundings pressure $p$ to eliminate any changes in $p^{\text {in }}$ associated with changes ot the particle radius $r$. These variables are interconnected via the YoungLaplace equation $p^{\text {in }}-p=2 f / r$.
}

$$
\mu^{\mathrm{b}}=\left(\frac{\partial G^{\mathrm{b}}}{\partial \mathrm{n}}\right)_{T, p}=G_{\mathrm{m}}^{\mathrm{b}} .
$$

Similarly the surface contribution can be expressed as

$$
\mu^{\mathrm{S}}=\left(\frac{\partial G^{\mathrm{S}}}{\partial n}\right)_{T, p^{\text {in }}}=\gamma\left(\frac{\partial A}{\partial n}\right)_{T, p^{\text {in }}}=\gamma V_{\mathrm{m}}\left(\frac{\partial A}{\partial V}\right)_{T, p^{\text {in }}}
$$

We consider here only isomorphic changes of a particle, e.g. the changes of particle volume without any shape changes, and so $(\partial A / \partial V)=2 / r$.

Thus

$$
\mu^{\mathrm{np}}=G_{\mathrm{m}}^{\mathrm{b}}+\frac{2 \gamma V_{\mathrm{m}}}{r} .
$$

The difference between the surface contribution to the molar Gibbs energy and chemical potential of $\mathrm{Au}$ nanoparticle of radius $r$ is shown in Figure 1.

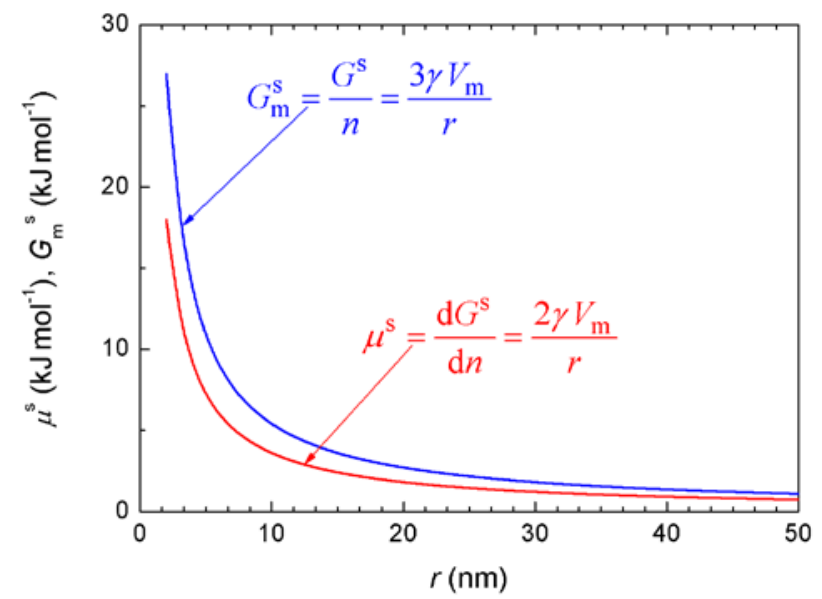

Figure 1. (Color online) The difference between surface contribution to molar Gibbs energy and chemical potential of Au nanoparticle of radius $r$ $\left(V_{\mathrm{m}}=10.6 \times 10^{-6} \mathrm{~m}^{3} \mathrm{~mol}^{-1}, \gamma=1.764 \mathrm{~J} \mathrm{~m}^{-2}\right)$

It follows from the above presented equations that both surface terms are correct but with a different meaning. Due to the fact, that equilibrium conditions are always derived from differential relations $\mathrm{d} G=0$ or $\mathrm{d} F=0$, the surface term with the multiplier 2 (eq. (13)) should be correctly used.

\section{Applications for Gas-liquid and Solid-liquid Equilibria}

Suppose a solid or liquid spherical nanoparticle (phase $\alpha$ ) of radius $r$ which is surrounded by a fluid phase (phase $\beta$ ). Let such a system be closed at a constant temperature $T$ and pressure of fluid phase $p$. The pressure $p^{\text {in }}$ within the condensed nanoparticle is, due to the surface stress, higher than the pressure of fluid phase. If the pressure dependence of the condensed phase molar volume is neglected the following relation should be satisfied at equilibrium $[20,21]$

$$
G_{\mathrm{m}, \alpha}^{\mathrm{b}}(T, p)+\frac{2 \gamma V_{\mathrm{m}, \alpha}}{r}=G_{\mathrm{m}, \beta}^{\mathrm{b}}(T, p) .
$$


This equation has the general form $F(T, p, r)=0$ and setting one of the variables $T, p$ or $r$, a dependence between the other two can be obtained.

Considering a liquid nanoparticle (nanodroplet) - vapor equilibrium at a constant temperature $T$ and pressure $p_{r}$ the well-known Kelvin equation should be simply derived from Eq. (14)

$$
\begin{gathered}
G_{\mathrm{m}, \mathrm{g}}^{\mathrm{b}}\left(T, p_{r}\right)-G_{\mathrm{m}, \mathrm{l}}^{\mathrm{b}}\left(T, p_{r}\right) \\
\doteq G_{\mathrm{m}, \mathrm{g}}^{\mathrm{b}}\left(T, p_{\infty}\right)+R T \ln \frac{p_{r}}{p_{\infty}}-G_{\mathrm{m}, \mathrm{l}}^{\mathrm{b}}\left(T, p_{\infty}\right)=\frac{2 \gamma_{\lg } V_{\mathrm{m}, \mathrm{l}}}{r} \\
\ln \frac{p_{r}}{p_{\infty}}=\frac{2 \gamma_{\lg } V_{\mathrm{m}, \mathrm{l}}}{R T r}
\end{gathered}
$$

where $p_{\infty}$ is the equilibrium pressure of the vapor above the plane surface and $p_{r}$ above the surface with curvature $1 / r$. Note that the difference $G_{\mathrm{m}, \mathrm{l}}\left(p_{r}\right)-G_{\mathrm{m}, \mathrm{l}}\left(p_{\infty}\right)$ has been omitted. Calculated vapor pressures of selected liquid metals at their melting points above spherical nanodroplets of radius $r$ according to the Kelvin equation (16) are shown in Figure 2.

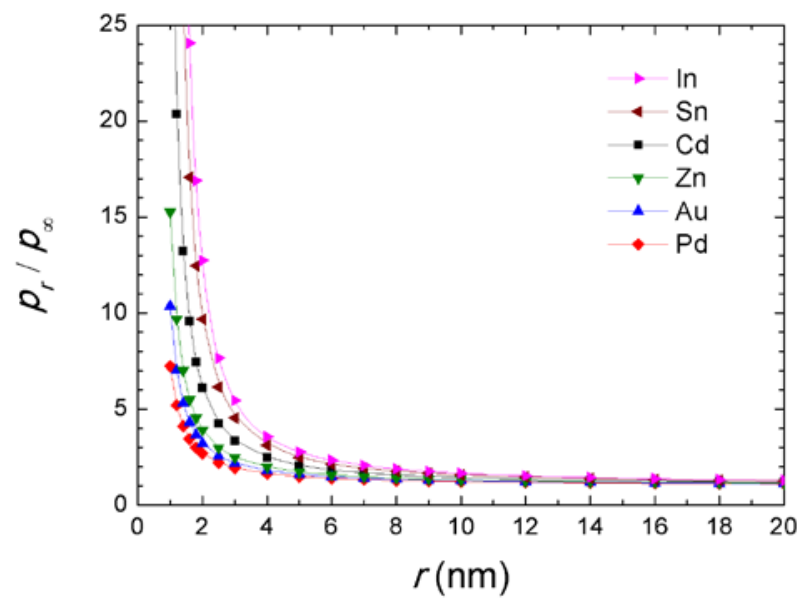

Figure 2. (Color online) Calculated vapor pressures of selected liquid metals at their melting points above spherical nanodroplets of radius $r$ according to the Kelvin equation (16)

Considering a solid nanoparticle - liquid phase (melt) equilibrium at a constant temperature $T_{\text {fus }, r}$ and pressure $p$, the Gibbs-Thomson equation should be derived in the form

$$
\begin{gathered}
G_{\mathrm{m}, \mathrm{l}}^{\mathrm{b}}\left(T_{\text {fus }, r}, p_{r}\right)-G_{\mathrm{m}, \mathrm{s}}^{\mathrm{b}}\left(T_{\text {fus }, r}, p_{r}\right) \\
\doteq \Delta_{\text {fus }} H_{\mathrm{m}}\left(1-\frac{T_{\text {fus }, r}}{T_{\text {fus }, \infty}}\right)=\frac{2 \gamma_{\mathrm{sl}} V_{\mathrm{m}, \mathrm{s}}}{r} \\
\frac{T_{\text {fus }, r}}{T_{\text {fus }, \infty}}=1-\frac{2 \gamma_{\mathrm{sl}} V_{\mathrm{m}, \mathrm{s}}}{\Delta_{\text {fus }} H_{\mathrm{m}} r} .
\end{gathered}
$$

$T_{\text {fus, } \infty}$ is the equilibrium melting temperature for a macroscopic solid and $T_{\text {fus, } r}$ for a spherical nanoparticle of radius $r$. Note that the temperature dependence of the enthalpy of melting has been omitted. Calculated melting temperatures of selected metals in the form of spherical nanoparticles of radius $r$ according to the Gibbs-Thomson equation (18) are shown in Figure 3.

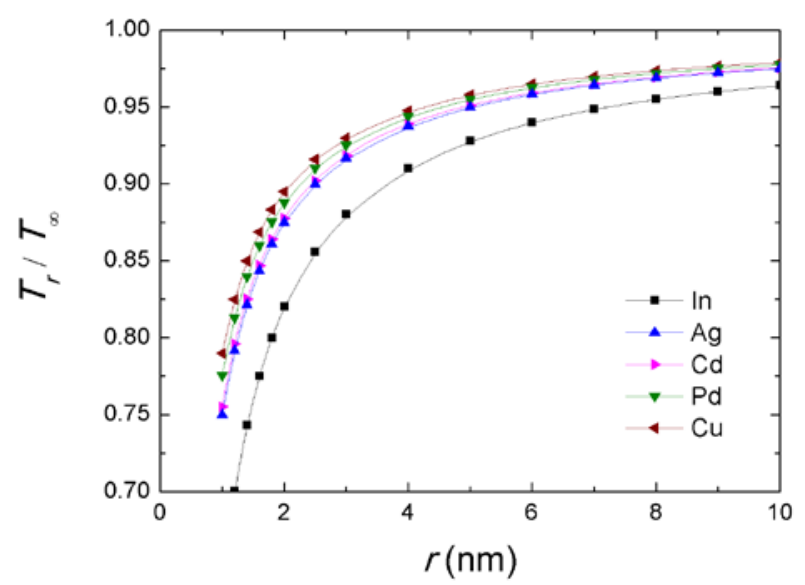

Figure 3. (Color online) Calculated melting temperatures of selected metals in the form of spherical nanoparticles of radius $r$ according to the Gibbs-Thomson equation (18)

\section{Conclusion}

In this article, a controversial issue in nanothermodynamics, namely the correct form of surface contributions to the molar Gibbs energy and chemical potential of single-component nanoparticles is explained. To better understand this difference it is convenient to use a formal approach based on the homogeneous functions formalism. Moreover, using the correct form of the equilibrium condition (the equality of chemical potentials) simple derivation of Kelvin and Gibbs-Thomson equations is presented.

\section{Acknowledgements}

This work was supported by Czech Science Foundation, grant No. 17-13161S.

\section{References}

[1] Guisbiers, G.; Mejia-Rosales, S.; Khanal, S.; Ruiz-Zepeda, F.; Whetten, R. L.; José-Yacaman, M. Gold-Copper Nano-Alloy, "Tumbaga", in the Era of Nano: Phase Diagram and Segregation. Nano Lett. 14. 6718-6726. 2014.

[2] Sim, K.; Lee, J. Phase stability of Ag-Sn alloy nanoparticles. J. Alloys Compounds 590. 140-146. 2014.

[3] Sopoušek, J.; Vřeštál, J.; Pinkas, J.; Brož, P.; Buršík, J.; Stýskalík, A.; Škoda, D.; Zobač, O.; Lee, J. Cu-Ni nanoalloy phase diagram Prediction and experiment. CALPHAD 45. 33-39. 2014.

[4] Kroupa, A.; Káňa, T.; Buršík, J.; Zemanová, A.; Šob, M. Modelling of phase diagrams of nanoalloys with complex metallic phases: application to Ni-Sn. Phys. Chem. Chem. Phys. 17. 28200-28210. 2015.

[5] Ghasemi, M.; Zanolli, Z.; Stankovski, M.; Johansson, J. Size- and shape-dependent phase diagram of In-Sb nano-alloys. Nanoscale 7. 17387-17396. 2015.

[6] Du, J.; Zhao, R.; Xue, Y. Effects of sizes of nano-copper oxide on the equlibrium constant and thermodynamic properties for the reaction in nanosystem. J. Chem. Thermodynamics 45. 48-52. 2012.

[7] Du, J.; Zhao, R.; Xue, Y. Thermodynamic properties and equilibrium constant of chemical reaction in nanosystem: An 
theoretical and experimental study. J. Chem. Thermodynamics 55. 218-224. 2012.

[8] Cui, Z.; Duan, H.; Li, W.; Xue, Y. Theoretical and experimental study: the size dependence of decomposition thermodynamics of nanomaterials. J. Nanopart. Res. 17. 321 (11 pp.). 2015.

[9] Li, W.; Cui, Z.; Duan, H.; Xue, Y. Effect of Nanoparticle size on the thermal decomposition thermodynamics in theory and experiment. Appl. Phys. A 122. 99 (12 pp.). 2016.

[10] Kaptay, G. The Gibbs Equation versus the Kelvin and the GibbsThomson Equations to Describe Nucleation and Equilibrium of Nano-Materials. J. Nanosci. Nanotechnol. 12. 2625-2633. 2012.

[11] Kaptay, G. Nano-Calphad: extension of the Calphad method to systems with nano-phases and complexions. J. Mater. Sci. 47. 8320-8335. 2012.

[12] Makkonen, L. Comments on "The Gibbs Equation versus the Kelvin and the Gibbs-Thomson Equations to Describe Nucleation and Equilibrium of Nano-Materials”. Adv. Sci. Focus 1. 367-368. 2013.

[13] Lee, J.; Sim, K.J., General equations of CALPHAD-type thermodynamic description for metallic nanoparticle systems. CALPHAD 44. 129-132. 2014.

[14] Guenther, G.; Guillon, O. Models of size-dependent nanoparticle melting tested on gold. J. Mater. Sci. 49. 7915-7932. 2014.
[15] Bajaj, S.; Haverty, M. G.; Arróyave, R.; Goddard III FRSC, W. A.; Shankare, S. Phase stability in nanoscale material systems: extension from bulk phase diagrams Nanoscale, 7. 9868-9877. 2015.

[16] Blinder, S.M. Mathematical method in elementary thermodynamics. J. Chem. Education 43. 85-92. 1966.

[17] Letellier, P.; Mayaffre, A.; Turmine, M. Nonextensive approach to thermodynamics: Analysis and suggestion, and application to chemical reactivity. J. Phys. Chem. B 108. 18980-18987. 2004.

[18] Letellier, P.; Mayaffre, A.; Turmine, M. Solubility of nanoparticles: nonextensive thermodynamics approach $J$. Phys.: Condens. Matter 19. 436229 (9 pp). 2007.

[19] Letellier, P.; Mayaffre, A.; Turmine, M. Melting point depression of nanosolids: Nonextensive thermodynamics approach. Phys. Rev. B 76. 045428 (8 pp). 2007.

[20] Cammarata, R.C. Generalized thermodynamics of surfaces with applications to small solid systems. in Solid State Physics. 61. 1-75. 2009.

[21] Leitner, J.; Sedmidubský, D. Thermodynamic equilibria in system with nanoparticles. in Thermal Physics and Thermal Analysis. Springer International Publishing, 2017. 385-402. 